\title{
All-Fiber Erbium Doped Fiber Laser Based on an Intracavity Polarizing Fiber Grating
}

\author{
Chengbo Mou, Zuxing Zhang, Zhijun Yan, Kaiming Zhou, Lin Zhang and Sergei Turitsyn \\ Aston Institute of Photonic Technologies, Aston University, Birmingham, B4 7ET, United Kingdom \\ Authore-mail address:moucl@aston.ac.uk
}

\begin{abstract}
Using Aston-made special design polarizing grating, we have implemented a stretchpulse mode locked erbium fiber laser. The laser has a simple and efficient all-fiber configuration with 90 fs output pulse duration and1.68 nJ pulse energy.

OCIS codes: (060.3735) Fiber Bragg gratings ; (140.3510) Lasers ,erbium; (140.4050) Mode locked lasers
\end{abstract}

\section{Introduction}

Mode locked ultrafast operation fiber lasers are vitally important tools in the contemporary science and industrial applications such as e.g. communication, sensing, imaging and micromachining and others. Passively mode locked fiber lasers are attractive due to their ease of fabrication, compact design, low cost and low power consumption. By balancing different effects such as self-phase modulation, gain saturation, group velocity dispersion (GVD) and high order dispersion, femtosecond pulses can be now routinely generated using passive mode locking. Various mode locking mechanisms responsible for pulse formation and shaping are exploited in a fiber laser cavity, including nonlinear polarization rotation (NPR) [1], semiconductor saturable absorber mirror(SESAM) [2], carbon nanotubes [3] and graphene [4]. Nonlinear dynamics is also very important and fiber lasers are now offering nice platform for studying new nonlinear lasing regimes such as similariton [5], dissipative soliton [6] and hybrid regimes [7].

To make the best use of the all-fiber structure of the fiber laser, the selection of a proper mode locker or mode locking mechanism is very critical. Polarizer is a critically important element of a fiber laser systems widely applied in NPR mode locking of fiber lasers. However, the standard bulk polarizers inherently break down the all-fiber structure of a fiber laser system while other type of fiber polarizers possess less robustness and high insertion loss. Polarizing fiber grating is a novel type of in-fiber polarizer device based on a $45^{\circ}$ tilted grating structure. In principle, it is very similar to a pile of plate polarizer which based on the Brewster angle therefore radiating out slight and preserving p-light for propagation [8]. With the benefits of low cost, all-fiber structure and low insertion loss, such device has found various applications as polarization dependent loss (PDL) equalizer, polarimeter and allfiber polarization filter. Such grating has also shown promising potential for the application of fiber lasers including CW single polarization fiber laser [9], soliton mode locked fiber laser [10] and dissipative soliton mode locked fiber laser [11]. In this report, using Aston-designed and fabricated polarizing grating we have presented a stretch pulse mode locked erbium doped fiber laser which demonstrates the shortest pulse duration generation through a fiber grating based ultrafast operation fiber laser. Simplicity and efficiency of implementation is a key feature of the demonstrated laser system.

\section{Polarizing Fiber Grating Fabrication and Characterization}

The polarizing fiber grating used in the experiment were inscribed in a conventional telecom fiber using the standard phase mask scanning technique and a $244 \mathrm{~nm}$ UV source from a $\mathrm{CW}$ frequency doubled $\mathrm{Ar}^{+}$laser. The fibers were hydrogen loaded at 150 bar and $80^{\circ} \mathrm{C}$ for two days prior to the UV inscription to further enhance the photosensitivity. The phase mask with a uniform period of $1800 \mathrm{~nm}$ was specially designed to have the fringe pattern tilted at $33.7^{\circ}$ with respect to the fiber axis, which would generate internal tilted index fringes at $45^{\circ}$ in the fiber core with a wide radiation response around the telecom window. The insertion loss and PDL of the polarizing grating was characterized through a commercial measurement kit (from LUNA system) incorporating a tunable laser showing the response of the grating in the range from $1525 \mathrm{~nm}$ to $1608 \mathrm{~nm}$. This measured spectral range was defined by the spectra range of the available tunable laser. Fig. 1(a) depicts the insertion loss of the grating within the measurement range, illustrating an average $\sim 1.7 \mathrm{~dB}$ insertion loss across the entire spectrum for $p$-light while a huge loss for $s$-light. This is in contrast to a standard fiber grating where no noticeable PDL will be observed. Fig. 1(b) shows the characteristic PDL of the polarizing fiber grating over a large wavelength range which covers the typical gain bandwidth of erbium doped fiber. It is obvious to see that the PDL has a wavelength dependency and the maximum PDL value at $1550 \mathrm{~nm}$ is $\sim 30 \mathrm{~dB}$. 

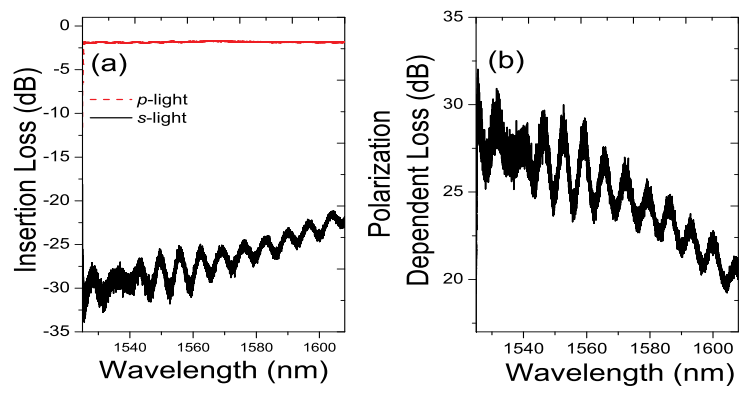

Fig.1 Measured (a) insertion loss of p-light and s-light; (b) PDL response of the polarizing grating

\section{Fiber laser Setup and Experimental Results}

The configuration of the polarizing grating based mode-locked erbium-doped fiber laser is depicted in Fig. 2. The laser consists of $\sim 1.2 \mathrm{~m}$ highly doped erbium-doped fiber (EDF) with nominal absorption coefficient of $\sim 80 \mathrm{~dB} / \mathrm{m}$ at $1530 \mathrm{~nm}$ and GVD $\beta_{2}=+66.1 \mathrm{ps}^{2} / \mathrm{km}$. The rest of the cavity consists of $\sim 0.4 \mathrm{~m} \mathrm{HI} 1060$ fiber with anomalous GVD $\beta_{2}=-7 \mathrm{ps}^{2} / \mathrm{km}$ and $\sim 2.6 \mathrm{~m}$ SMF28 with $\beta_{2}=-22.8 \mathrm{ps}^{2} / \mathrm{km}$. The net GVD of the laser cavity is $\sim 0.013 \mathrm{ps}^{2}$ which is slightly normal therefore showing our laser works in the stretch pulse regime. One polarization independent optical isolators (OIS) are used to ensure single direction oscillation. The fiber laser is pumped through a wavelength division multiplexer (WDM) from a grating stabilized $975 \mathrm{~nm}$ laser diode. A commercial laser diode driver and controller are employed to stabilize the pump laser. Two in-fiber polarization controllers (PC1 \& PC2) are located before and after the polarizing grating to perform NPR mode locking. Two 90:10 fiber couplers are employed to tap $10 \%$ of laser power out of the cavity where one is for laser outputs after the gain fiber and the other is located before the WDM for studying the pulse dynamics within the cavity.

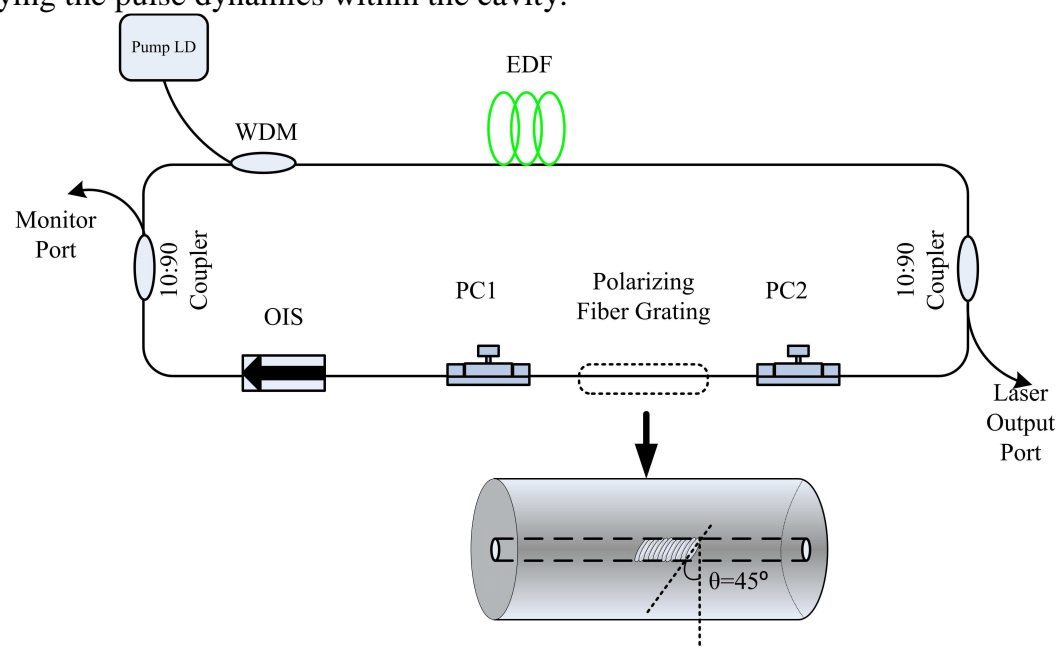

Fig.2 schematic of mode-locked ultrashort pulse fiber laser with polarizing fiber grating. The inset is sketch of the polarizing fiber grating.

By properly adjusting PC1 and PC2 in the system, stable mode-locked pulses can be easily achieved. The optical pulses duration have been examined using a commercial optical autocorrelator. The lead fiber of the laser output port was cut gradually to about $2.3 \mathrm{~m}$ in order to optimize the obtained pulse duration. Fig. 3(a) shows the full-width half-maximum (FWHM) of autocorrelation trace is $127 \mathrm{fs}$ corresponding to pulse duration of $\sim 90 \mathrm{fs}$ when Gaussian fit is assumed. The estimated pulse duration at output point of the laser cavity is 1.2 ps. Fig. 3(b) shows the optical spectrum profile centered at $1575 \mathrm{~nm}$ for both output port and monitor port with a spectral bandwidth at FWHM of $\sim 54 \mathrm{~nm}$, thus giving a time-bandwidth product of $\sim 0.58$, indicating the compressed Gaussian pulse is a little beyond the transform limited. This is possibly owing to the third-order dispersion of the laser cavity and the outside compression fiber. With the measured pulse duration and spectra width of the compressed pulse, the calculated pulse duration at the output point of the laser cavity is 2.4 ps. A typical pulse train is shown in Fig. 3(c) 
with a $\sim 21 \mathrm{~ns}$ interval between two adjacent pulses, giving a repetition rate of $\sim 47.8 \mathrm{MHz}$. The output pulse power is $8 \mathrm{~mW}$, which corresponds to the output energy of $\sim 1.68 \mathrm{~nJ}$. Fig. 3(d) shows the RF spectrum of laser at fundamental frequency showing a high signal to noise ratio of $\sim 65 \mathrm{~dB}$. The laser is stable under laboratory condition for $>24$ hours. Although many modern mode-locked lasers provide higher energy pulses with shorter durations, here we have realized the genuine all-fiber mode-locked ultrashort laser using the polarizing fiber grating. It is noted that the output spectra from the different ports have similar spectral profile and width, except distinct power difference. Since the net GVD is slightly normal, the laser works in a dissipative stretch-pulse regime.
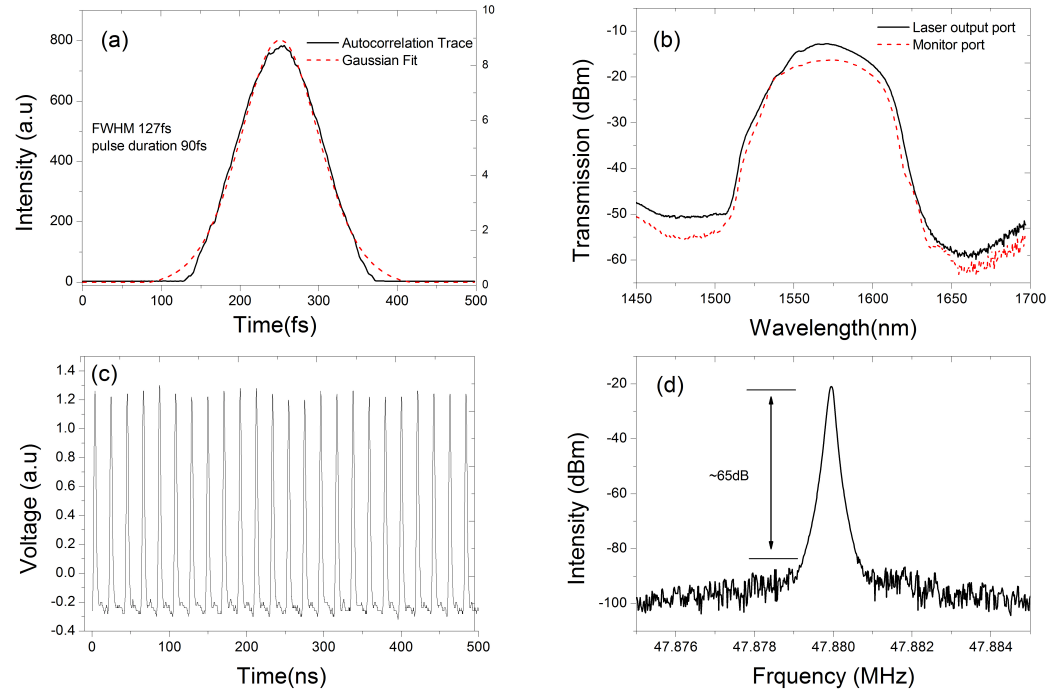

Fig.3 Measured characteristics of laser (a) autocorrelation trace (black line) and its Gaussian fitting (red dotted line), (b) optical spectra of both output (black line) and monitor (red dotted line) ports; (c) pulse trains; (d) RF spectrum.

\section{Conclusions}

In conclusion, we have successfully demonstrated a stretch pulse mode locked erbium doped fiber laser based on a polarizing fiber grating. The unique polarization property of the grating guarantees a compact and integrated allfiber structure. To the best of our knowledge, this is the shortest pulse generated from a fiber grating mode locked erbium doped fiber laser.

\section{References}

[1] K.Tamura, H. A. Haus, and E. P. Ippen, "Self-Starting additive pulse mode-locked erbium fiber ring laser," Electron. Lett. 28, 2226-2228 (1992).

[2] U. Keller, K. J. Weingarten, F. X. Kartner, D. Kopf, B. Braun, I. D. Jung, R. Fluck, C. Honninger, N. Matuschek, and J. A. Ausder,

"Semiconductor saturable absorber mirrors (SESAM's) for femtosecond to nanosecond pulse generation in solid-state lasers," IEEE J. Sel. Top. Quant. 2, 435-453 (1996).

[3] S. Y. Set, H. Yaguchi, Y. Tanaka, and M. Jablonski, "Ultrafast fiber pulsed lasers incorporating carbon nanotubes," IEEE J. Sel. Top. Quant. 10, 137-146 (2004)

[4] H. Zhang, Q. L. Bao, D. Y. Tang, L. M. Zhao, and K. Loh, "Large energy soliton erbium-doped fiber laser with a graphene-polymer composite mode locker," Appl. Phys. Lett. 95, 141103 (2009).

[5] F. Ö. Ilday, J. R. Buckley, W. G. Clark, and F. W. Wise, "Self-similar evolution of parabolic pulses in a laser," Phys. Rev. Lett. 92, 213902 (2004)

[6] P. Grelu and N. Akhmediev, "Dissipative solitons for mode-locked lasers," Nature Photon. 6, 84-92 (2012)

[7] B. Öktem, C. Ülgüdür, and F. Ö. Ilday, "Soliton-similariton fibre laser," Nature Photon. 4, 307-310 (2010)

[8] K. M. Zhou, G. Simpson, X. F. Chen, L. Zhang, and I. Bennion, "High extinction ratio in-fiber polarizers based on 45 degrees tilted fiber Bragg gratings," Opt. Lett. 30, 1285-1287 (2005)

[9] C. B. Mou, K. M. Zhou, L. Zhang, and I. Bennion, "Characterization of 45 degrees-tilted fiber grating and its polarization function in fiber ring laser," J. Opt. Soc. Am. B 26, 1905-1911 (2009)

[10] C. B. Mou, H. Wang, B. G. Bale, K. M. Zhou, L. Zhang, and I. Bennion, "All-fiber passively mode-locked femtosecond laser using a 45 degrees-tilted fiber grating polarization element," Opt. Express 18, 18906-18911 (2010)

[11] X. L. Liu, H. S. Wang, Z. J. Yan, Y. S. Wang, W. Zhao, W. Zhang, L. Zhang, Z. Yang, X. H. Hu, X. H. Li, D. Y. Shen, C. Li, and G. D. Chen, "All-fiber normal-dispersion single-polarization passively mode-locked laser based on a 45 degrees-tilted fiber grating," Opt. Express 20, 19000-19005 (2012) 